\title{
Effects of Stellar Collisions on Star Cluster Evolution and Core Collapse
}

\author{
Sourav Chatterjee, John M. Fregeau \\ and Frederic A. Rasio
}

Department of Physics and Astronomy, Northwestern University, Evanston, IL 60208, USA email: s-chatterjee@northwestern.edu, fregeau@northwestern.edu, rasio@northwestern.edu

\begin{abstract}
We systematically study the effects of collisions on the overall dynamical evolution of dense star clusters using Monte Carlo simulations over many relaxation times. We derive many observable properties of these clusters, including their core radii and the radial distribution of collision products. We also study different aspects of collisions in a cluster taking into account the shorter lifetimes of more massive stars, which has not been studied in detail before. Depending on the lifetimes of the significantly more massive collision products, observable properties of the cluster can be modified qualitatively; for example, even without binaries, core collapse can sometimes be avoided simply because of stellar collisions.
\end{abstract}

Keywords. scattering, stellar dynamics, methods: numerical, (stars:) blue stragglers, (Galaxy:) globular clusters: general

\section{Introduction}

In dense stellar systems like massive young clusters, galactic centers, and old globular clusters (GC), the high densities of stars can give rise to many direct, single-single physical collisions, in addition to collisions mediated by dynamical interactions of binaries (Fregeau et al. 2004). In these systems stellar evolution can also be significantly modified through physical collisions. For example, in young star clusters, collisions can give rise to runaway growth of merger products, producing many exotic stellar populations, such as intermediate-mass black holes (Gürkan et al. 2006; Trenti 2006). The cores of typical old Galactic GCs can also attain high enough densities so that most core stars undergo collisions during their lifetime (Hills \& Day 1976; Fregeau et al. 2004). These collisions not only change the evolution of individual stars, but the increased stellar masses also change the overall GC properties like the core radius $\left(r_{c}\right)$ and the half-mass radius $\left(r_{h}\right)$. Moreover, at least some of the observed exotic stellar populations in dense clusters, like blue straggler stars (BSS) (Fekadu et al. 2007; Sills et al. 2001; Sills et al. 1997), and compact binaries like ultracompact X-ray binaries (UCXB), are likely created through collisions (Lombardi et al. 2006).

A dense cluster of stars naturally evolves towards eventual core collapse through relaxation. At their present ages, most Galactic GCs are expected to have collapsed cores. However, observations show that the measured values of $r_{c} / r_{h}$ for most Galactic GCs are higher than predicted by theoretical models (Vesperini \& Chernoff 1994). Many scenarios have been proposed to explain this apparent discrepancy. For example, the core can be supported against deep collapse by dynamically extracting the binding energy of hard primordial binaries (Trenti et al. 2007; Fregeau \& Rasio 2007). However, it is hard to explain most of the high observed values of $r_{c} / r_{h}$ in Galactic GCs purely through this "binary burning" process. Other mechanisms to halt core collapse like ejection of stellar mass BHs from the core (Mackey et al. 2007) or stellar captures by a central 
Table 1. Initial Conditions of Simulated GCs

\begin{tabular}{cccccccc}
\hline & Model & IMF & $\mathrm{N}$ & $n_{\text {binary }}$ & $r_{c}(\mathrm{pc})$ & radius $(\mathrm{pc})$ & $\rho_{c}\left(M_{\odot} / p c^{3}\right)$ \\
\hline Cases 1,2 & Plummer & Single-Mass & $10^{6}$ & 0 & 0.3 & 0.85 & $10^{6}$ \\
Case 3 & King, $w_{0}=6$ & Kroupa $\left(0.1-2.0 M_{\odot}\right)$ & $10^{6}$ & 0 & 0.87 & 2.89 & $1.7 \times 10^{4}$ \\
\hline
\end{tabular}

intermediate-mass black hole (Trenti et al. 2007; Trenti 2006) have also been discussed at this meeting (see contributions by Mackey and Trenti in this volume). It has also been suggested that $r_{c} / r_{h}$ could simply keep increasing over long timescales in clusters having fairly long relaxation times via ongoing mass segregation (Merritt et al. 2004).

Here we study stellar collisions as a possible mechanism for supporting clusters against core collapse. In a regime where collisions are important, they can produce many stars significantly more massive than those in the background population. Thus the subsequent evolution of collision products will be much faster than for normal stars in the cluster. Although the stellar evolution and observable properties of collision products have been extensively studied before (Sills et al. 2001; Sills et al. 1997), the feedback effects of collisions on the overall dynamical evolution of GCs has received less attention. The shorter evolution timescales of massive collision products may support the core against collapse even without any primordial binaries or other mechanisms for energy production, simply via indirect heating through stellar evolution mass loss (Goodman \& Hernquist 1991; Goodman \& Hut 1989; Lee 1987).

Using $N$-body simulations, we have begun studying numerically how collisions and the subsequent evolution of collision products can alter the overall properties of GCs. We use the Northwestern group's Hénon-type Monte-carlo code, which provides a detailed, star-by-star representation of clusters with up to $N \sim 10^{6}-10^{7}$ stars (Fregeau \& Rasio 2007; Joshi et al. 2001; Joshi et al. 2000). In $\S 2$ we will first present two simple limiting cases, bracketing reality and illustrating the dramatic changes in global cluster properties depending on how the evolution of collision products is treated in the models. We also present the evolution of a more realistic GC model with a conventional "rejuvenation" prescription for determining the lifetimes of collision products. We discuss the implications of our study and planned future work in $\S 3$.

\section{Results}

All simulations shown here use $N=10^{6}$ stars initially. We assume totally conservative collisions with the interaction cross section given by the usual "sticky sphere" approximation. There is no hydrodynamic mass loss during the collisions. However, mass loss occurs through normal evolution of the stars via (instantaneous) compact object formation at the end of the main sequence (MS) lifetime. The collision products can be significantly more massive and hence evolve faster, enhancing the overall stellar mass loss rate. Determining theoretically the true MS lifetime or the effective age of a collision product is a hard task. These quantities depend sensitively on the amount of hydrogen mixed into the core of the collision product as a result of the collision. This in turn can be highly variable because the mixing depends on the details of the collision kinematics. To avoid these uncertainties, in our study we use three very simple prescriptions for determining the effective ages and the remaining MS lifetimes of collision products. Case 1: Infinite lifetime for all stars, including the collision products. Case 2: Zero lifetime for the collision products. Other stars have infinite lifetime, unless they collide. Case 3: A more realistic "rejuvenation" prescription for determining the age and MS lifetime of the 



Figure 1. a) Evolution of $r_{c} / r_{h}$. Solid line: no collisions. Dotted line: case 1, where all stars have infinite lifetime (including collision products). Dashed line: case 2, where collision products have zero lifetime. In case 2 collapse is avoided through mass loss due to evolution of the collision products. b) Evolution of $E$ (top panel) and the core radius (bottom panel). The steepest slope in the $E$ evolution curve corresponds to core collapse. Onset of collapse can also be seen directly in the $r_{c}$ evolution plot. c) Evolution of $E$ (top panel), evolution of $r_{c}$ in physical units (middle panel), and Evolution of the average mass contained in three Lagrange radii $(0.01,0.1,0.9)$ of the GC (bottom panel) for case 3. d) Evolution of $r_{c} / r_{h}$ for case 3. e) Histogram of $r_{c} / r_{h}$ of all Galactic GCs with known $r_{c}$ and $r_{h}$ values.

collision products: we assume that stars burn $\mathrm{H}$ linearly on the MS so the effective age of a collision product is uniquely determined by the total amount of $\mathrm{H}$ coming from the progenitors. The age and the MS lifetime of each collision product is determined by the following simple equation, based on Hurley et al. (2002),

$$
t=\frac{t_{M S}}{M}\left(\frac{t_{1} M_{1}}{t_{M S 1}}+\frac{t_{2} M_{2}}{t_{M S 2}}\right) .
$$

Here, $t_{i}, M_{i}$, and $t_{M S i}$ are the age, mass, and the MS lifetime of parent star $i$, while $t$, $M$, and $t_{M S}$ are for the collision product. Note that the coefficient on the right hand side of Eq. 2.1 is different from the one used in Hurley et al. (2002). We use Eq.2.1 so that two zero age main sequence (ZAMS) stars collide to produce a ZAMS star, whereas two stars close to their MS turn-off collide to produce another star close to its turn-off.

\subsection{Limiting cases: Single mass Plummer model (cases 182 )}

The initial cluster properties are listed in Table1. Fig. 1a shows the evolution of $r_{c} / r_{h}$ for the two limiting cases as well as the case without collisions for comparison purposes. Clearly, conservative collisions make the cluster more bound manifested by the faster collapse of the core than the no collision case in case 1. In the other limiting case, case $\mathbf{2}$, on the onset of collapse the densities reach a very high value increasing the collision rate. The mass loss after the collisions can stop the collapse and reach a steady core radius with a low rate of further collisions. This mechanism can be better illustrated by Fig. 1b. We define energy $E$ in such a way that it remains constant unless mass is lost from the cluster. The onset of collapse can be seen in the simultaneous decrease of $r_{c}$ and the increased slope of the $E$ evolution curve. 

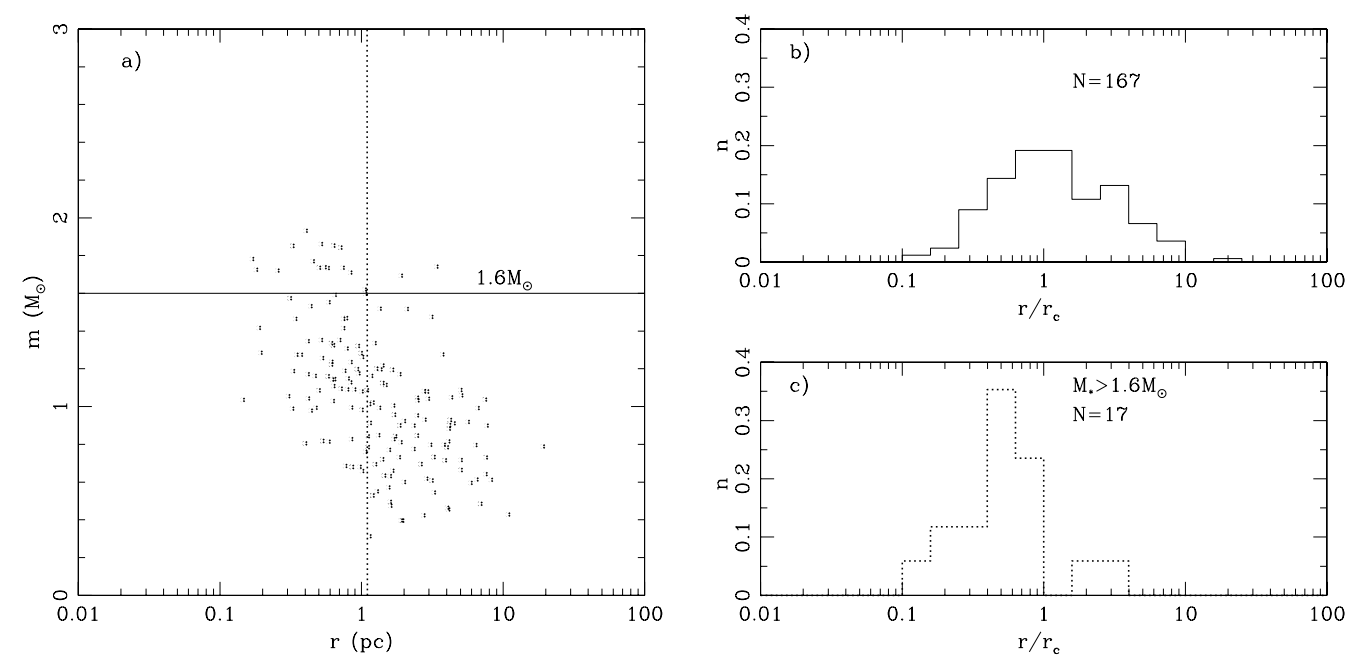

Figure 2. a) Position vs mass scatter plot for all collision products still at their MS life at 14 Gyr. Two times the present age turn-off mass $\left(1.6 M_{\odot}\right)$ is shown as a horizontal solid line to guide the eye. The position of the core is shown with the vertical dotted line. b) Histogram showing the positions of the same population as in $(a)$. c) Histogram showing the positions of the collision products still in their MS life at present age having masses $\geqslant 1.6 M_{\odot}$.

\subsection{More realistic case: rejuvenation-based prescription; King sphere, $w_{0}=6$ (Case 3 )}

The initial cluster properties are listed in Table1. Here we first evolve the cluster with only stellar evolution for $10^{7}$ years, so that the very massive stars evolve and disappear from the system. We then switch on dynamics.

Till a little more than 1 Gyr $E$ remains constant, indicating no collisions (Fig. 1c). Sometime between $1 \& 2 \mathrm{Gyr}$, the core starts to collapse (Fig. 1c) reducing the core radius and increasing the average mass in the core region. Thus collision rate increases significantly increasing rate of mass loss. This is manifested in the sudden steep increase in $E$. Thus through mass loss core collapse is avoided and $r_{c} / r_{h}$ approaches a steady value (Fig 1d). Fig 1d,e shows that, even with the simple rejuvenation prescription, the final value of $r_{c} / r_{h}$ compares well with the observed values for most Galactic GCs. The slow continued increase of $E$ at later times comes as normal stars as well as some low mass collision products go off the MS and disappear.

Fig. 2a shows the positions of the collision products that are still in their MS life at 14 Gyr. Most of these collision products are contained within the core. BSS candidates are most easily identified above $\sim 2$ times the turn-off mass of the cluster. The more massive subset of collision products in our simulated cluster $\left(M \geqslant 1.6 M_{\odot}\right)$ are mostly found inside the core (Fig. 2c) (apart from only 2 just outside the core).

\section{Discussion}

Although determination of stellar evolution after a collision is a subject of continuing research, the effects of collisions on cluster dynamics have not been studied in detail previously. This is a first report of an ongoing systematic study on this effect. Using typical initial conditions for old GCs and simple assumptions for rejuvenation we show that collisions between single stars not only alter the stellar properties and produce exotic 
stellar populations like some of the BSS, they also affect the overall GC properties. Most importantly, collisions can support the core of a cluster against collapse in typical old Galactic GCs. Even with our simple but reasonable assumptions, the values of $r_{c} / r_{h}$ obtained for our simulated GCs compare well with the observed $r_{c} / r_{h}$ values for Galactic GCs. Furthermore, we obtain a population of BSS candidates contained within the core, also consistent with observations (Leigh et al. 2007). Note that we do not find any BSSs well outside the core, consistent with the current understanding that those BSSs are most likely formed via primordial binary mergers (Mapelli et al. 2006).

We have adopted many extreme simplifications for this first look at the problem. For example, at this stage of our study, once a star evolves off the MS, it is removed from the simulation, leaving no remnant. Since remnants are normally only a few percent of the total progenitor mass, we expect that this approximation will not affect the overall GC properties significantly, so far as the increase in energy is concerned from mass loss. However, some remnants from very massive stars at a very young age can remain in the cluster and sink into the core through dynamical friction. Dynamical interactions, including collisions, of these massive remnants can also alter the core properties of the GCs in certain regimes (Mackey et al. 2007; Trenti et al. 2007; Trenti 2006). Another possibly important effect left out of this study for now is the role of primordial binaries (Fregeau \& Rasio 2007; Fregeau et al. 2004). On the one hand, the presence of primordial binaries will increase the rate of collisions through resonant encounters (Fregeau et al. 2004), on the other hand, binaries will provide further support of a cluster against collapse and hence may prevent the core from reaching high enough densities for significant collisions.

\section{Acknowledgements}

This work was supported by NASA Grants NNG04G176G and NNG06GI62G.

\section{References}

Fekadu, N., Sandquist, E. L., \& Bolte, M. 2007, ApJ 663, 277

Fregeau, J. M. \& Rasio, F. A. 2007, ApJ 658, 1047

Fregeau, J. M., Cheung, P., Potegies Zwart, S. F., \& Rasio, F. A. 2004, MNRAS 352, 1

Goodman, J. \& Hernquist, L. 1991, ApJ 378, 637

Goodman, J. \& Hut, P. 1989, Nature 339, 40

Gürkan, M. A., Fregeau, J. M., \& Rasio, F. A. 2006, ApJ 640, L39

Hills, J. G. \& Day, C. A. 1976, Aplett 17, 87

Hurley, J. R., Tout, C. A., \& Pols, O. R. 2002, MNRAS 329, 897

Joshi, K. J., Nave, C. P., \& Rasio, F. A. 2001, ApJ 550, 691

Joshi, K. J., Rasio, F. A., \& Portegies Zwart, S. 2000, ApJ 540, 969

Lee, H. M. 1987, ApJ 319, 801

Leigh, N., Sills, A., \& Knigge, C. 2007, ApJ 661, 210

Lombardi, Jr., J. C., Proulx, Z. F., Dooley, K. L., Theriault, E. M., Ivanova, N., \& Rasio, F. A. 2006, ApJ 640, 441

Mackey, A. D., Wilkinson, M. I., Davies, M. B., \& Gilmore, G. F. 2007, MNRAS 379, L40

Mapelli, M., Sigurdsson, S., Ferraro, F. R., Colpi, M., Possenti, A., \& Lanzoni, B. 2006, MNRAS 373,361

Merritt, D., Piatek, S., Portegies Zwart, S., \& Hemsendorf, M. 2004, ApJLett 608, L25

Sills, A., Faber, J. A., Lombardi, Jr., J. C., Rasio, F. A., \& Warren, A. R. 2001, ApJ 548, 323

Sills, A., Lombardi, Jr., J. C., Bailyn, C. D., Demarque, P., Rasio, F. A., \& Shapiro, S. L. 1997, ApJ 487, 290

Trenti, M. 2006, ArXiv Astrophysics e-prints 12040

Trenti, M., Ardi, E., Mineshige, S., \& Hut, P. 2007, MNRAS 374, 857

Vesperini, E. \& Chernoff, D. F. 1994, ApJ 431, 231 